\title{
Conceptual model of productivity bot for smart construction planning
}

\author{
Donatas Kavaliauskas ${ }^{1}$, Leonidas Sakalauskas ${ }^{2}$ \\ ${ }^{1}$ Data Science and Digital Technologies, Vilnius University, Vilnius, Lithuania \\ ${ }^{2}$ Fundamental Sciences, Vilnius Gediminas Technical University, Vilnius, Lithuania \\ E-mail: 'donatas.worshipper@gmail.com (corresponding author)
}

\begin{abstract}
One of the most important tasks in modern construction is to build a building according to the desired time schedule. This requires a timetable for the construction stages processes. The schedule is also easy to adapt and to plan the renovation of a building or maintenance works. Without a good schedule site manager cannot effectively handle construction area processes. The solution to this problem is the proposed productivity bot concept based on metaheuristic algorithms. Metaheuristic algorithms allow to improve the construction planning process schedules compared with conventional planning methods and equipment. The results of testing with construction planning data has shown that the metaheuristic algorithm achieved the main improvements during the first planning optimization stages. The proposed concept is expected to present results that are close to the optimal timetable and surpass classical methods for scheduling. Productivity bots act as a software engine complementing with the organization of construction or automation functionality. Productivity bot is designed to manage building processes. It is intended for automated planning of construction stages schedules according to construction technologies.
\end{abstract}

Keywords: structural mechanics and optimization, building information modeling, operational research, maintenance and renovation of buildings.

\section{Introduction}

At present, the construction sector is in the greatest lack of precision in construction planning. Often, construction lasts longer than the deadlines due to improperly calculated weather conditions or incorrectly distributed workloads, or other factors (Awasthi, 2016). In this way, construction companies lose potential capital for failing to make a timely project. This is the potential for the company to grow and create new jobs. To avoid this, a system is being designed which, with the help of artificial intelligence, will plan construction work, maintenance, and renovation of buildings. It is predicted that construction work schedules will be up to $15 \%$ higher than the standardized timetables.

\section{Mainframe}

Productivity robots as a software tool engine, complementing production organization or automation functionality. When a robot is connected to a software platform, it increases the benefit of the tool you are already using. Productivity robots can be included in various applications (Raman \& Tok, 2018; Ballesteros-Pérez, Thor Smith, Gwen LloydPapworth, \& Cooke, 2018).

The construction productivity robot is designed for managing construction project stages. It is designed for product flow scheduling for automated planning of construction plans schedule. The suggested system the construction productivity bot provides benefits like:

- The program automatically updates schedules in real time.

- Learning from historical data.

- Using artificial intelligence to provide intelligent solutions or intelligent information (Lebeuf, 2013).

We suggest supplementing the traditional construction plan with the features of artificial intelligence and additionally include side effects that may affect the implementation of the plan. It is shown in Figure 1.

(C) 2019 Authors. Published by VGTU Press. This is an open-access article distributed under the terms of the Creative Commons Attribution (http://creativecommons.org/licenses/by/4.0/) License, which permits unrestricted use, distribution, and reproduction in any medium, provided the original author and source are credited. 


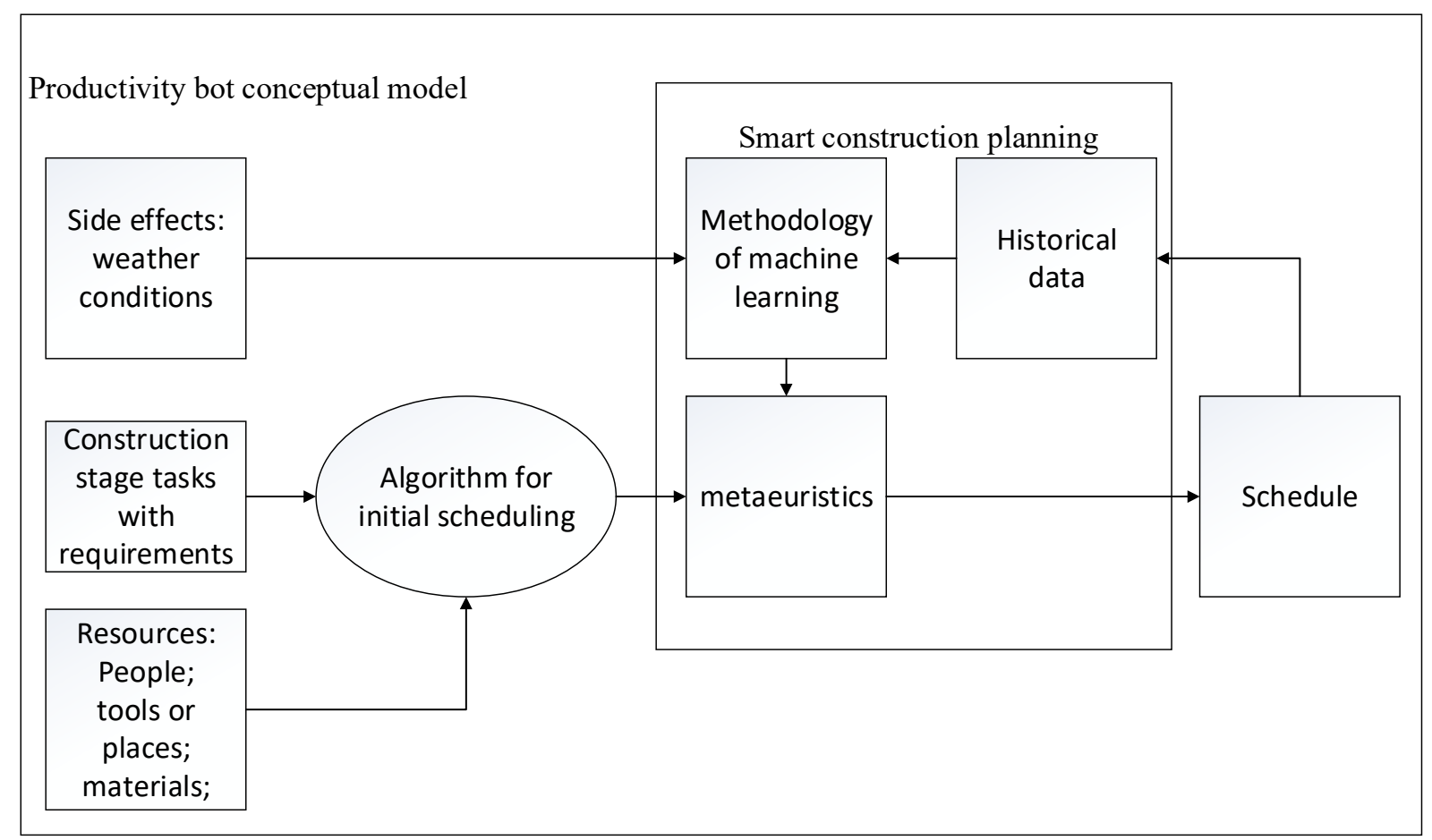

Figure 1. Productivity bot conceptual model

\section{Technology}

Deep learning neural networks will be used to create the product. The intelligent software productivity robot that we are about to create will stand out in the market from other products with artificial intelligence features like learning and decision making. The Productivity Robot will build adaptive intellectual rules using historical learning scheduling techniques using deep learning neural networking methodology. These rules will allow you to calibrate metaheuristics algorithm parameter sets in real-time, predict and analyze critical construction situations, and propose solutions to problems. Such robot learning will significantly shorten the timeframe for future scheduling and increase the quality of schedules. At the same time, the prototype that is being developed will require less administrative costs because the smart software productivity robot will operate autonomously.

The project will develop a smart software productivity robot that will be able to forecast and analyze critical construction situations of construction planning schedules in real time and to propose solutions to problems. Also, in real time, calibrate construction planning parameters and make construction schedules according to the class of the optimization tasks being solved. Existing methods and equipment for calibration and scheduling of metaheuristics algorithm parameters take 1 to 48 hours depending on the technical capabilities and complexity of the tasks. The real need for real-time management of all modern production management conditions is a huge need. Methods of calibration of metaheuristics algorithms for production planning, analysis of schedules, forecasting critical construction situations will be based on methods of deep learning neural networks.

\section{Methaeuristics}

The simulated annealing algorithm for solving formal combinatorial optimization tasks was introduced in 1983 by Kirkpatrik, and other authors. The pioneers of this model were Metropolis, Rosenbluth and others (1953). This algorithm is based on the simulation of energy processes taking place in systems composed of a large number of particles. Computer studies of the simulated annealing algorithm may reveal the features of applying metaheuristic algorithms to scheduling optimization (Kirkpatrick, Gelatt, \& Vecchi, 1983).

The main idea of the algorithm is to simulate the annealing (tempering) of the body, which is also indicated by the name of the algorithm. When simulating system annealing, the system is initially given a high temperature, which is gradually reduced until the system goes into a stationary state (the steel grows).

The accuracy of the response depends on the choice of temperature. If we choose too high a temperature, we can "catch" (not found) the optimal solution. On the other hand, if you choose a low temperature, the algorithm will work in one place and find an only local optimum. So the choice of temperature is an important factor for this algorithm.

The simulated annealing algorithm is easy to apply for optimizing construction process planning. We plan to use this algorithm to create an optimization engine to deliver better results for the proposed system. Because the problem of the problem is the stochastic type algorithms of the NP complexity is the right choice to solve the problem. In order 
for SA to produce more accurate results, it is necessary to calibrate the algorithm parameters for the task class. Machine learning algorithms can be utilized for parsing parameters, as a soft learning neural network. This method makes it possible to use historical data and calibrate parameters.

\section{Weather probability estimation}

A solid construction project plan should allow for unforeseen weather conditions and there should be measures in place to mitigate the effects. For example, heavy rain can prevent employees from working with electrical appliances due to a safety requirement, which may stop work for a day. Another example, extreme heat can also cause discoloration in the paint. Bricks can become too dry in hot weather (Ballesteros-Pérez et al., 2018). This will decrease a brick's ability to adhere to the mortar paste and thus create weak masonry work. In order to assess the impact of weather on the schedule of the construction plan, a model will be developed that uses this weather forecast to evaluate this criterion. The module will determine the impact using historical data and actual weather forecast. The weather forecast estimate will depend on the time span of the current date. The further the date is planned, the lower the estimate will be for the weather forecast. At the same time, weather forecasting prediction and real weather at the predicted date will be stored as training data. In this way, using the machine learning algorithm, the system will make a more accurate assessment of the impact on the quality of construction schedules.

\section{Construction resources}

There are various stages in construction that need to be planned. For example, conducting communications, founding foundations or piles, spilling walls, building a roof structure and others. Resources need to be planned for all these stages. Resources can be divided into peoples, tools or places and materials. Everyone has their own compotes that are qualified to perform. For this reason, a bricklayer cannot perform the work of a welder because it is not his specialty. Thus, employees need to plan their work according to their competencies, but other tasks can be assigned to it if necessary. Another aspect of each construction company is the limited amount of resources as electrical appliances. Because it is also a planned resource that is directly related to the employees in the construction project planning system, they are also included as resources.

\section{Conclusions}

It is expected that the intelligent software productivity robot that is being developed will allow automation of construction plans. It is also expected to provide very high quality production scheduling in real time. It is expected that the timetable created for construction companies will save up to $15 \%$ of the time with the available resources. Improving the planned construction project time will make it easier for construction companies to compete in the market with companies from other countries and to increase capital. The prototype being created will select the most appropriate metaheuristics algorithm according to the class of the problem to be addressed, calibrating the metaheuristics algorithm parameters, which will ensure the quality of the schedule and speed of scheduling. The system will have the option of manually selecting the scheduling method and assigning parameters to it. Also, the intelligent software productivity robot will have the ability to compare production scheduling with artificial intelligence with standard algorithm results.

The third resource is building materials. The construction stages require materials to be ordered in time for a continuous construction process. It is necessary to plan the construction materials in such a way that they should not be stored, and the warehousing of other materials requires special conditions in order not to affect the climate.

\section{References}

Awasthi, A. (2016). Optimization of NP-hard scheduling problems by developing timing algorithms and parallelization (Dissertation). Carl von Ossietzky Universität Oldenburg.

Ballesteros-Pérez, P., Thor Smith, S., Gwen Lloyd-Papworth, J., \& Cooke, P. (2018). Incorporating the effect of weather in construction scheduling and management with sine wave curves: application in the United Kingdom. Construction Management and Economics, 36(12), 666-682. https://doi.org/10.1080/01446193.2018.1478109

Kirkpatrick, S., Gelatt, C. D., \& Vecchi, M. P. (1983). Optimization by simulated annealing. Science, 220(4598), 671-680. https://doi.org/10.1126/science.220.4598.671

Lebeuf, C. R. (2013). A taxonomy of software bots: towards a deeper understanding of software bot characteristics (Master thesis). University of Victoria. Retrieved from https://dspace.library.uvic.ca/bitstream/handle/1828/10004/Lebeuf_Carlene_MASc_2018.pdf?sequence=1\&isAllowed=y

Raman, A., \& Tok, W. H. (2018). A developer's guide to bulding AI applications. O’Reilly Media, Inc. 